\title{
Photodynamic therapy with aminolevulinic acid: a novel therapeutic target in the management of gastrointestinal malignancies
}

\begin{abstract}
Surgery combined with chemoradiotherapy is the mainstay for the management of gastrointestinal malignancies. Ultimate goal is to eliminate locoregional recurrences, to prolong survival and to prevent morbidity and mortality associated with traditional treatment modalities in management of gastrointestinal malignancies. ,In this respect, Photodynamic Therapy (PDT) seems to be a good alternative treatment option for gastrointestinal malignancies because of its promising results in precancerous, cancerous and noncancerous lesions. Studies support the complete response with PDT in recurrent and advanced cases with gastrointestinal malignancies. Aminolevulinic acid (ALA) is a heme precursor leading to accumulation of the photosensitizer protoporphyrin IX (PpIX) in tissues that makes ALA useful target for PDT. Thus, PDT using ALA seems to be promising novel, advanced, an efficient, convenient approach in management of patients with gastrointestinal malignancies. This review highlights the principles of action mechanism of PDT with ALA, applications in gastrointestinal malignancies and limitations and its future directions in this field.
\end{abstract}

Keywords: 5-aminolevulinic acid, Photodynamic therapy, Gastrointestinal cancers, Gastric cancer, Colorectal cancer, Hepato-pancretico-biliary cancer
Volume 8 Issue 3 - 2017

\author{
Emel Canbay, ${ }^{1,2,3,4}$ Egesinan Torun, ${ }^{5}$ Bahar \\ Canbay Torun, ${ }^{6}$ Satoshi Wakama, ${ }^{2,3}$ Yutaka \\ Yonemura ${ }^{1,2,3}$ \\ INPO HIPEC, Turkey \\ ${ }^{2} \mathrm{NPO}$ to support Peritoneal Surface Malignancy, Japan \\ ${ }^{3}$ Peritoneal Disease Center, Kishiwada Tokushukai Hospital, \\ Japan \\ ${ }^{4}$ Department of General Surgery, Biruni University, Turkey \\ ${ }^{5}$ Department of Internal Medicine, Istanbul University, Turkey \\ ${ }^{6}$ Department of General Surgery, Istanbul University, Turkey
}

Correspondence: Emel Canbay MD, PhD, Center for Peritoneal Surface Malignancies, Istanbul 34365, Turkey, Tel 00 (90) 212296 0909, Fax 00(90) 2122336950 ,

Email drecanbay@gmail.com

Received: February 28, 2017 | Published: August 15, 2017

\section{Introduction}

The concept of photodynamic therapy (PDT) has initiated after Raab, a medical student and his teacher Von Tappeiner discovered that certain dyes could cause cell death in the presence of light source. Even though, first clinical trials were conducted in dermatological disease, PDT did not have an enough attention until 1970s. ${ }^{2}$ Then, PDT has increasingly studied as therapeutic modality in precancerous, cancerous and non-cancerous lesions in humans.

Here, we describe basic principles of action of PDT with ALA, applications in gastrointestinal malignancies and limitations and its future directions in this field.

\section{Principles of photodynamic therapy and ala as a photo synthesizer}

PDT requires three essential components: a photosensitizer, a light source and oxygen. The photosensitizer is a molecule that localizes to the target cells or tissues. When the photosensitizer is exposed to specific wavelengths of light, it becomes activated from the ground state to excited state and causes the production reactive oxygen species (ROS) including singlet oxygen (1O2) which trigger the destruction of tumour cells via apoptosis and necrosis. ${ }^{3}$

Recently, aminolevulinic acid (ALA) has been used as photosensitizer for PDT. ${ }^{4,5}$ ALA is first committed intermediate in the heme synthesis pathway (Figure 1). ${ }^{6} \mathrm{~A}$ heme synthesis pathway has two rate limiting enzymes that are, ALA synthase and ferrocheletase. ${ }^{7}$ Ferrochelatase is responsible for converting protoporphyrin IX (PPIX) into heme. Exogenous ALA administration saturates ferrochelatase, resulting in PPIX accumulation. ${ }^{5}$ ALA is not a photosensitizer but its end product PpIX is photoreactive substance.

Preferentially uptaken of ALA is resulted with PpIX accumulation in malignant cells. ${ }^{5}$ Excess accumulation of PpIX may occur because of low activity of ferrochelatase which catalyses the insertion of an iron atom into PpIX or higher activity of hydroxymethylbilane synthase in malignant cells. ${ }^{8}$ Beside this, there is an accumulating evidence suggests that membrane transport systems of ALA and porphyrin play very important role in accumulation of PpIX., ${ }^{9,10}$

The wavelength of the light source must correspond to a maximal absorption peak of the photo synthesizer in order to achieve proper cell death. A light source with high fluence rate and capable of delivering a large dose of light in a short time is also important. ${ }^{11}$

The presence of oxygen in a tumour cell is also important for success of PDT, since oxygen is required for photochemical reactions to occur, and hypoxic regions tend to be not responsive to photo synthesizer. ${ }^{12}$

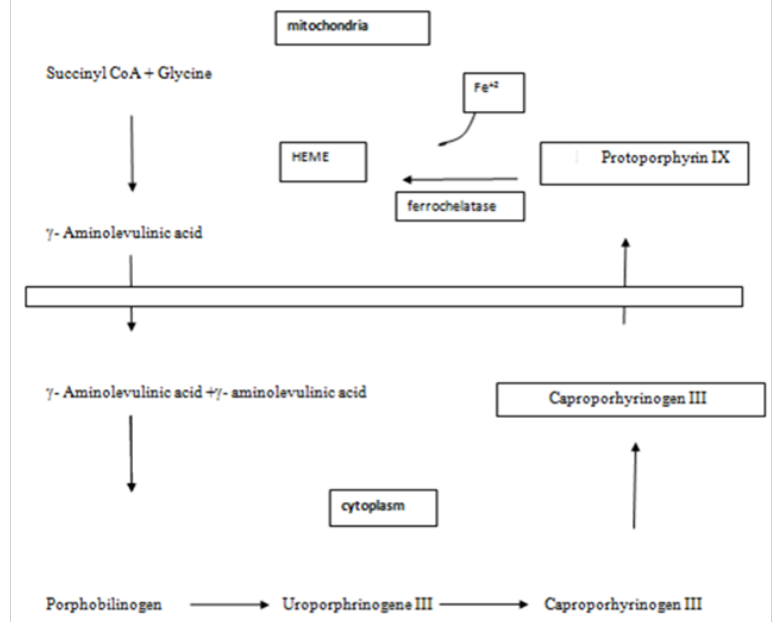

Figure I Heme synthesis. 
New generations of ALA as a photosensitizer with spectrums of absorption at higher wavelengths such as $650-740 \mathrm{~nm}$ have allowed deeper tissue penetration and more extensively studied in worldwide. Methyl ester derivative of ALA is approved for treatment of basal cell carcinoma of the skin and actinic keratosis. ${ }^{13,14}$ ALA is administered topically or systemically via oral, intravenous and intradermal routes. When ALA administered systemically, it distributes to virtually all tissues preferentially in liver and tissues with elevated metabolic rates. ${ }^{15}$ Side effects of systemic and oral administrations are nausea, vomiting, photosensitization in skin and urinary frequency, alterations of liver function, hypotension in 4-6 hours and resolving within 24 hours. ${ }^{16,17}$ Currently, 5-ALA is only cleared for use dermatologic, malignant glioma, urogenital applications, ${ }^{5}$ but is used for gastrointestinal and other systemic applications. ${ }^{18}$

\section{ALA photodynamic therapy (ALA-PDT) in gastrointestinal cancers and peritoneal carcinomatosis}

\section{ALA-PDT in upper gastrointestinal system}

The use of ALA-PDT in superficial and early lesions of upper gastrointestinal system diseases shows great promise as an alternative to invasive techniques. Evidence for these options is discussed below. Premalignant lesions such as Barrett's oesophagus with high grade dysplasia are theoretically ideal for treatment with PDT. ${ }^{19}$ These are superficial and large mucosal areas easily accessible for light. Dysplasia may arise in the setting of Barrett's oesophagus and that can lead to the development of adenocarcinoma. A phase I study showed that high grade dysplasia can be eradicated $89 \%$ with free from cancer rate $96 \%$ after 36 months follow up in patients treated with red light after $60 \mathrm{mg} / \mathrm{kg}$ ALA. ${ }^{20}$ The first study of ALA-PDT in the human gastrointestinal tract organs; oesophagus, duodenum and colorectal tumours was described as a pilot study in $1995 .{ }^{21}$ They reported that ALA-PDT is promising technique for the treatment of small tumours located to mucosa such as Barrett's oesophagus. However, in a phase II study demonstrated the poor response to ALA-PDT in respect for residual disease and progression of disease with recurrence ${ }^{22}$ Dunn and Lovat. ${ }^{23}$ systematically reviewed and synopsis was given from the literature for PDT-ALA for treatment of dysplasia in Barret's oesophagus. They reported that ALA-PDT is both safe and effective for the treatment of dysplasia and early cancer in Barret's oesophagus. Further studies are required to demonstrate the comparative effectiveness of PDT-ALA in the treatment of oesophageal and cardia lesions.

Studies investigating the role of ALA-PDT in gastric cancer are very limited. Both reports in vivo and in vitro were from China. ${ }^{24,25}$ ALA-PDT was reported to be an effective and promising therapy for gastric cancer following in vitro study on human gastric cancer cells ${ }^{24}$ and in vivo study with human gastric cancer cell lines injected to nude mice skin. ${ }^{25}$ A clinical experience of using ALA in combination with in chemo-immune-hyperthermia in patients with Stage IV gastric cancer was reported from Japan. ${ }^{26}$ They conclude that ALA can exert good palliation in combination with other therapies in late stages of gastric cancer. Hino et al. ${ }^{27}$ studied ALA-PDT using light emitting diodes (LED) light in different wavelength in a mouse model of peritoneal dissemination of gastric cancer. They reported ROS generation and cytotoxic effects of ALA-PDT were the highest at $410 \mathrm{~nm}$ and lowest at $635 \mathrm{~nm}$. Differences in light source indicate that optimal wavelength is one of the essential parameters in development of strategy with ALA-PDT. Again, ALA was found to be feasible and safe in biopsyproven early gastric carcinoma without visible lesions.$^{28}$ Even studies these suggested great promise regrettably were not followed by randomized clinical trials. Beside these studies, researchers have a great effort to understand ALA specific accumulation in cancer cells to increase effectiveness of ALA-PDT in vitro gastric cancer cell line. ${ }^{10}$ They have found that higher expression of peptide transporter PEPT1 (ALA influx transporter) and low expression of ATP-binding cassette transporter (ABCG2) (porfirin efflux transporter) determined ALAinduced PpIX production and photosensitivity in vitro.

Beside this, Porfimer Sodium has been used as a photo sensitizer in treatment of 113 patients with esophageal cancer after local failure following chemo radiotherapy. ${ }^{29}$ Complete remission rate was $58.4 \%$ (66/113) and 5-year progression free survival was $22.1 \%$ with overall survival was $35.9 \%$. Treatment related death was $1.8 \%$ in recurrent cases of esophageal cancer. Porfimer sodium is also curative option for superficial cases with esophageal cancer. ${ }^{30,31}$

\section{ALA-PDT in small bowel cancers}

ALA-PDT has been also applied to duodenal and ampulla vateri tumours. $^{21}$ and patients with polyps in duodenum due to familial adenomatosis polys. ${ }^{32}$ that indicating promising results. However, further studies are required to optimize the ALA-PDT.

\section{ALA-PDT in hepato-pancreatico biliary cancers}

ALA-PDT studies in hepatocellular carcinoma have been mostly limited to in vitro studies in cultured cell lines and in vivo murine studies. The accumulation of PpIX after administration of ALA was higher in tumoural tissue than that in non tumoural tissue in hepatocellular cancer model. ${ }^{33}$ ALA-PDT has been found to be decreasing tissue oxygenation 10 -fold in rat liver. ${ }^{34}$ ALA-PDT has been found to be yielded with apoptosis in $70 \%$ of HepG2 cells following 18 hours of ALA incubation. ${ }^{35}$ ALA-PDT has been resulted also cell death in hepatoblastoma cell lines. ${ }^{36}$ PDT within pancretico-biliary system cancers has been extensively studied with comparatively little focus to ALA-PDT. Tumour growth was significantly decreased in in vitro and in vivo studies with ALA-PDT and with other photo synthesizers. ${ }^{37,38}$ Little attention to ALA-PDD in hepatopancreaticobiliary cancers may due to a report showed no effect in bile duct cancer in $2001 .^{39}$ However, hexyl aminolevulinic acid form successfully expressed higher concentration of PpIX in chlangiocarcinoma cell lines expressing $\mathrm{H}+$ peptide co transporter PEPT1 suggesting PEPT1 and other forms of ALA may be very effective in PDT approach of cholangiocarcinoma. ${ }^{40}$

\section{ALA-PDT in colorectal cancer}

ALA-PDT again understudied in colorectal cancer. PDT with other photosynthesizer was used in the treatment of villous adenoma ${ }^{41}$ and polyps $^{42}$ of colorectum. More recently, it has been reported that ALAPDT using blue and white LED light demonstrated greater antitumour effects in vitro and in vivo. ${ }^{43}$ It has been reported that selective inhibition of $\mathrm{H}+$-coupled aminoacid transporter PAT1 and increased $\mathrm{H}+$-coupled di/tripeptide transporter PEPT1 could contribute to ALAPDT as an underlying mechanism in accumulation of PpIX in colon cancer patients. ${ }^{44}$

\section{ALA-PDT in peritoneal malignancies}

ALA-PDT has not been studied in peritoneal surface malignancies even ALA guided diagnosis has been reported in primary peritoneal serous carcinoma ${ }^{45}$ and peritoneal malignancies of ovarian cancers. ${ }^{46}$ Peritoneal malignancy management using CRS and HIPEC has been evolved in past years. ${ }^{47}$ Promising results in management of peritoneal malignancies with PDT have been reported from the 
most of the previous studies carried out using photofrin in peritoneal surface malignancies with severe side effects such as capillary leak syndrome. ${ }^{48,49}$

Several studies have shown that ALA-mediated photodynamic diagnosis (PDD) is a promising tool for diagnosing peritoneal metastasis in gastric cancer. ALA-mediated PDD was used in addition to diagnostic laparoscopy for 52 patients with advanced gastric cancer. Of these patients, peritoneal metastasis has been detected in 5 of $24(20.8 \%)$ patients with no evidence of peritoneal metastasis using ALA-mediated PDD. ${ }^{50}$

Photodynamic therapy can be even preventive effect to development of peritoneal metastasis. Talaporfin as a second generation photosensitizer mediated photodynamic therapy (PDT) has been reported to have a more accumulation in metastatic nodules and an anti-tumor effects resulted with longer survival rates in mice model developed peritoneal metastases. ${ }^{51}$

\section{Combination of photodynamic therapy with immunotherapy}

Even though cancer immunotherapy has shown promising results, high proportion of patients may relapse or given limited response. PDT can reduce tumor burden and increase the secretion of tumor antigen from the cancer cells. Combination of PDT with immunotherapy might have better outcomes in cancer therapy. Indeed, PDT can be combined with tumor specific immunotherapy with long peptide vaccination. ${ }^{52}$

Further studies will be required for effect of ALA-PDT in peritoneal surface malignancies.

\section{Five year view}

Studies of PDT with ALA in other gastrointestinal malignancies and peritoneal carcinomatosis are limited to few phase I and II clinical and in vitro/in vivo studies. Photodynamic Therapy (PDT) represents the method of choice for some of the diseases including minimally invasive therapeutic procedure to treat oesophageal cancer with high cure rates, low side effects. Increasing uptake of photosensitizer into the cells with appropriate light source and wavelength need to be investigated will improve the effects of PDT in cancerous cells. Furthermore, combination of PDT with immunotherapy has shown promising results in the treatment of advanced cancers.

\section{Key issues}

1. Studies of Photodynamic Therapy (PDT) with alanine (ALA) are aimed to improve oncologic treatment option

2. ALA may serve for better target therapy and better patient outcomes due to its ability to selectively accumulates in cancerous cells

3. Studies of PDT with ALA are limited in gastrointestinal cancers and peritoneal carcinomatosis

4. In vitro and in vivo studies significantly vary with regard to protocols, duration and outcome measurements

5. Technical aspects such as delivering light source, increased sensitivity and specific activity of photosensitizers and dosing strategies need to be clarified with further studies

6. Well controlled studies are necessary to determine whether PDT with ALA may add value to traditional treatment approaches
7. PDT with ALA deserves further investigation to elucidate ALA's role in the surgical therapies

\section{Expert commentary}

ALA-PDT is considered to be a novel and promising antitumor strategy. It promise to be targeted accurately and selectively in earlier and advances lesions. It can be used repeatedly with comparatively limited side effects. Disadvantages of ALA-PDT are limited tissue penetration, not selective to tumour cells and tissues. Beside this, its potential has not been fully demonstrated. Furthermore, its applications and in combination with other approaches are also not defined. The fact that PDT definitely reduces morbidity compare to surgery and in combination with chemo radiotherapy. PDT also can be repeated without compromising the efficacy.

Further studies including the cellular mechanism of ALA-PDT and using nanotechnology to increase the sensitivity and specificity of photo sensitizers (third generation of photosensitizer, etc.) and to develop accurate dose and light source for selective treatment certainly will develop our understanding and produce more effective therapy for not only gastrointestinal but also for other malignancies.

\section{Acknowledgments}

None.

\section{Conflicts of Interest}

Author declares there are no conflicts of interest.

\section{Funding}

None.

\section{References}

1. Moan J, Peng Q. An outline of the history of PDT. In:Patrice T (Ed.), Photodynamic Therapy. UK: The Royal Society of Chemistry; 2003. p. $3-5$

2. Dougherty TJ, Kaufman JE, Goldfarb A, et al. Photoradiation therapy for the treatment of malignant tumors. Cancer Res. 1978;38(8):26282635 .

3. Celli JP, Spring BQ, Rizvi I, et al. Imaging and photodynamic therapy:mechanisms, monitoring, and optimization. Chem Rev. 2010;110(5):2795-2838.

4. Brown SB, Brown EA, Walker I. The present and future role of photodynamic therapy in cancer treatment. Lancet Oncol. 2004;5(8):497-508.

5. Krammer B, Plaetzer K. ALA and its clinical impact:from bench to bedside. Photochem Photobiol Sci. 2008;7(3):283-289.

6. Kennedy JC, Pottier RH. Endogenous protoporphyrin IX, a clinically useful photosensitizer for photodynamic therapy. $J$ Photochem Photobiol. 1992;14(4):275-292.

7. Hunter GA, Ferreira GC. Molecular enzymology of 5-aminolevulinate synthase, the gate keeper of heme biosynthesis. Biochim Biophys Acta. 2011;1814(11):1467-1473.

8. Peng Q, Warloe T, Berg K, et al. 5-aminolevulinic acid based photodynamic therapy. clinical research and future chalenges. Cancer. 1997;79(12):2282-2308.

9. Rodriguez L, Batlle A, Di Venosa G, et al. Study of the mechanisms of uptake of 5-aminolevulinic acid derivatives by PEPT1 and PEPT 2 transporters as a tool to improve photodynamic therapy of tumors. Int $J$ Bochem Cell Biol. 2006;38:1530-1539. 
10. Hagiya Y, Endo Y, Yonemura Y, et al. Pivotal role of peptide transporter PEPT1 and ATP-binding cassette (ABC) transporter ABCG2 in 5aminolevulinic acid (ALA)-based photocytotoxicity of gastric cancer cells in vitro. Photodiagnosis Photodyn Ther. 2012;9(3):204-214.

11. Juzeniene A, Juzenas P, Ma LW, et al. Effectiveness of different light sources for 5-aminolevulinic acid photodynamic therapy. Lasers Med Sci. 2004;19(3):139-149.

12. Chen B, Pogue BW, Zhou X, et al. Effect of tumor host microenvironment on photodynamic therapy in a rat prostate tumor model. Clin Cancer Res. 2005;11(2):720-727.

13. Fayter D, Corbett M, Heirs M, et al. A systematic review of photodynamic therapy in the treatment of precancerous skin conditions, Barret's oesophagus and cancers of the biliary tract, brain, head and neck, lung, oesophagus and skin. Health Technol Assess. 2010;14(37):1-288.

14. Morton CA, Brown SB, Collins S, et al. Guidelines for topical photodynamic therapy:report of workshop of the British photodermatology Group. Br J Dermatol. 2002;146(4):552-567.

15. Egger NG, Motamedi M, Pow-Sang M, et al. Accumulation of porphyrins in plasma and tissues of dogs after delta aminolevulinic acid administration:implications for photodynamic therapy. Pharmacology. 1996;52(6):362-370.

16. Chung IW, Eljamel S. Risk factors for developing oral 5-aminolevulinic acid-induced side effects in patients undergoing fluorescence guided resection. Photodiagn Photodyn Ther. 2013;10(4):362-367.

17. Waidelich R, Hofstetter A, Stepp H. Clinical experience with 5aminolevulinic acid and photodynamic therapy for refractory superficial bladder cancer. J Urol. 2001;165(6):1904-1907.

18. Messmann H, Geisler M, Gross U, et al. Influence of a haematoporphyrin derivative on the protoporphyrin IX synthesis and photodynamic effect after 5-aminolaevulinic acid sensitization in human colon carcinoma cells. Br J Cancer. 1997;76(7):878-883.

19. Qumseya BJ, David W, Wolfsen HC. Photodynamic Therapy for Barrett's Esophagus and Esophageal Carcinoma. Clin Endosc. 2013;46(1):30-37.

20. Mackenzie GD, Dunn JM, Selvasekar CR, et al. Optimal conditions for successful ablation of high grade dysplasia in Barrett's oesophagus using aminolevulinic acid photodynamic therapy. Lasers in Medical Science. 2009;24(5):729-734.

21. Regula J, MacRobert AJ, Gorchein GA, et al. Photosensitization and photodynamic therapy of oesophageal, duodenal, and colorectal tumours using 5-aminolevulinic acid induced protoporphyrin IX - a pilot study. Gut. 1995;36(1):67-75.

22. Peters F, Kara M, Rosmolen W, et al. Poor results of 5-aminolevulinic acid-photodynamic therapy for residual high-grade dysplasia and early cancer in Barrett' esophagus after endoscopic resection. Endoscopy. 2005;37(5):418-424.

23. Dunn J, Lovat L. Photodynamic therapy using 5-aminolevulinic acid for the treatment of dysplasia in Barrete's oesophagus. Expert Opinion Pharmacother. 2008;9(5):851-858.

24. Huang ZH, Zhou GJ, Yu JL, et al. Effect of photodynamic therapy with 5 -aminolevulinic acid on human gastric cancer cells in vitro. $J$ South Med. 2006;26:255-257.

25. Zhou GJ, Huang ZH, Yu JL, et al. Effect of 5-aminolevulinic acidmediated photodynamic therapy on human gastric cancer xenografts in nude mice in vivo. Zhonghua Wei Chang Wai Ke Za Zhi. 2008;11:580 583.

26. Saito M, Yano K, Kaigaki T, et al. A patient with scirrhous stomach cancer treated with combination of hyperthermitherapy and 5aminolevulinic acid (ALA). Anticancer Res. 2013;33(7):2957-2963.

27. Hino H, Murayama Y, Nakanishi M, et al. 5 -aminolevulinic acid mediated photodynamic therapy using light emitting diodes of different wavelengths in a mouse model of peritoneal disseminated gastric cancer. J Surg Res. 2013;185(1):119-126.

28. Rabenstein T, May A, Gossner L, et al. Invisible gastric carcinoma detected by random biopsy:long term results after photodynamic therapy. Endoscopy. 2008;40(1):899-904.

29. Hatogai K, Yano T, Kojima T, et al. Salvage photodynamic therapy for local failure after chemoradiotherapy for esophageal squamous cell carcinoma. Gastrointest Endosc. 2016;83(6):1130-1139.

30. Nakamura T, Fukui H, Shirakawa K, et al. Photodynamic therapy of superficial esophageal cancer with a transparent hood. Gastrointest Endosc. 2004;60(1):120-124.

31. Tanaka T, Matono S, Nagano T, et al. Photodynamic therapy for large superficial squamous cell carcinoma of the esophagus. Gastrointest Endosc. 2011;73(1):1-6.

32. Mlkvy P, Messmann H, Debinski H, et al. Photodynamic therapy for polyps in familial adenomatous polyposis- a pilot study. Eur J Cancer. 1995;31(7-8):1160-1165.

33. Otake M, Nishiwaki M, Kobayashi Y, et al. Selective accumulation of ALA-induced PpIX and photodynamic effect in chemically induced hepatocellular carcinoma. Br J Cancer. 2003;89(4):730-736.

34. McIlroy BW, Curnow A, Buonaccorsi G, et al. Spatial measurement of oxygen levels during photodynamic therapy using time-resolved optical spectroscopy. J Photochem Photobiol B. 1998;43(1):47-55.

35. Yow CM, Wong CK, Huang Z, et al. Study of the efficacy and mechanism of ALA-mediated photodynamic therapy on human hepatocellular carcinoma cell.al. Liver Int. 2007;27(2):201-208.

36. Bergmann F, Stepp H, Metzger R, et al. In vitro and in vivo evaluation of photodynamic techniques for the experimental treatment of human hepatoblastoma and neuroblastoma:preliminary results. Pediatr Surg Int. 2008;24(12):1331-1333.

37. Pereira S. Photodynamic therapy for pancreatic and biliary cancer:the United Kingdom experience. J Natl Compr Canc Netw. 2012;10:S48S51.

38. Samkoe KS, Chen A, Rizvi I, et al. Imaging tumor variation in response to photodynamic therapy in pancreatic cancer xenograft models. Int J Radiat Oncol Biol Phys. 2010;76(1):251-259.

39. Zoepf T, Jakobs R, Rosenbaum A, et al. Photodynamic therapy with 5-aminolevulinic acid is not effective in bile duct cancer. Gastrointest Endosc. 2001;54(6):763-766.

40. Chung CW, Kim CH, Lee HM, et al. Aminolevulinic acid derivatives based photodynamic therapy in human intra- and extrahepatic cholangiocarcinoma cells. Eur J Pharm Biopharm. 2013;85(3):503-510.

41. Loh CS, Bliss P, Brown SG, et al. Photodynamic therapy for villous adenomas of the colon and rectum. Endoscopy. 1994;26(2):243-246.

42. Nakamura T, Fukui H, Ishii Y, et al. Photodynamic therapy with polypectomy for rectal cancer. Gastrointest Endosc. 2003;57(2):266269.

43. Hatakeyama T, Murayama Y, Komatsu S, et al. Efficacy of 5aminolevulinic acid mediated photodynamic therapy using light emitting diodes in human colon cancer cells. Oncol Rep. 2013;29(3):911-916.

44. Anderson CM1, Jevons M, Thangaraju M, et al. Transport of the photodynamic therapy agent 5 -aminolevulinic acid by distinct $\mathrm{H}+-$ coupled nutrient carriers coexpressed in the small intestine. J Pharmacol Exp Ther. 2010;332(1):220-228.

45. Canbay E, Ishibashi H, Sako S, et al. Photodynamic detection and management of intraperitoneal spreading of primary peritoneal papillary serous carcinoma in a man: report of a case. Surg Today. 2014;44(2):373-377. 
46. Liu Y, Endo Y, Fujita T, et al. Cytoreductive Surgery Under Aminolevulinic Acid-Mediated Photodynamic Diagnosis Plus Hyperthermic Intraperitoneal Chemotherapy in Patients with Peritoneal Carcinomatosis from Ovarian Cancer and Primary Peritoneal Carcinoma: Results of a Phase I Trial. Ann Surg Oncol. 20104;21(13):4256-4262.

47. Yonemura Y, Canbay E, Endou Y, et al. Peritoneal cancer treatment. Expert Opin Pharmacother. 2014;15(5):623-636.

48. Hahn SM, Fraker DL, Mick R, et al. A phase II trial of intraperitoneal photodynamic therapy for patients with peritoneal carcinomatosis and sarcomatosis. Clin Cancer Res. 2006;12(8):2517-2525.

49. Cengel KA, Glatstein E, Hahn SM. Intraperitoneal photodynamic therapy. Cancer Treat Res. 2007;134:493-514.
50. Kishi K, Fujiwara Y, Yano M, et al. Diagnostic laparascopy with 5aminolevulinic-acid-mediated photodynamic diagnosis enhances the detecteion of peritoneal micrometastases in advanced gastric cancer. Oncology. 2014;87(5):257-265.

51. Tsujimoto H, Morimoto Y, Takahata R, et al. Photodynamic therapy using nanoparticle loaded with indocyanine green for experimental peritoneal dissemination of gastric cancer. Cancer Sci. 2014;105(12):1626-1630.

52. Kleinovink JW, Van Driel PB, Snoeks TJ, et al. Combination of Photodynamic therapy and specidic Immunotherapy efficiently eradicates established tumors. Clin Cancer Res. 2016;22(6):1459-1468. 